\title{
The Application of Single Use Bioreactors for the Production of a Carotenoids Mix, Mainly Torularhodin
}

\author{
ALINA MIHALCEA ${ }^{1}$, ADRIAN ONU ${ }^{1}$, ANA AURELIA CHIRVASE ${ }^{3 *}$, CAMELIA UNGUREANU² \\ ${ }^{1}$ National Institute of Research and Development for Microbiology and Immunology Cantacuzino, 103 Splaiul Independentei , \\ 050096, Bucharest, Romania \\ 2University Politehnica of Bucharest, 1-7, 1 Polizu Str., 011061, Bucharest, Romania \\ 3Romanian Society of Bioengineering and Biotechnology, 1 Polizu Str., 011061, Bucharest, Romania
}

\begin{abstract}
The lab scale CellMaker Single Use Bioreactor made by Cellexus and BIOSTAT CultiBagRM Single Use Bioreactor made by Sartorius were tested versus a conventional Bioengineering reactor in order to increase the carotenoidic pigments concentration, mainly torularhodin concentration. For further research with the same yeast it is to consider both: the medium composition with phosphate addition, but also the Single Use Bioreactor Cellexus to get the highest torularhodin concentration in the carotenoids mix.
\end{abstract}

Keywords: carotenoids, torularhodin, Rhodotorula rubra, single use bioreactor

Due to applications of carotenoids as antioxidants, antimicrobial, antitumoral, antiaging agents and provitamin $A$, the carotenoids market will increase to 1,400 millions dollars in 2019 with a rate of $3.5 \%$ per year. It is $\beta$-caroten mostly, but also lutein, astaxanthin, lycopene, and canthaxanthin, the pigments that are industrially produced. Because in the case of production of pigments from plants there are some problems regarding seasonal and geographic variability that cannot be controlled and the chemical synthesis generates hazardous wastes for the environment, the microbial production of carotenoids shows great interest and safety to use.

Torularhodin, found in red yeasts such as Rhodotorula spp. and Sporobolomyces ruberrimus [1-7], is considered as an important carotenoid for protection against oxidative stress, being one of the few carotenoids with a carboxylic group. When $\beta$-carotene and torularhodin were added at the same molar concentration, torularhodin more effectively quenched singlet oxygen than $\beta$-carotene [8]. The mentioned yeasts produced a mix of mainly torularhodin, but also torulene, and $\beta$-carotene. These carotenoids are beneficial as precursors of vitamin A, antiaging, and may also prevent certain types of cancer and enhance the immune system [9-13]. These facts make the mix of these three carotenoids, mainly torularhodin, an important research topic, but the production and use of them is still in development stage.

The research demonstrated untill now that the $\beta$ carotene, torularhodin and torulene may be produced in liquid cultures, on a wide range of substrates, and are no growth associated bioproducts; as they are intracellular bioproducts there is a lot of information about the extraction methods comprising the cells mechanical, chemical or enzymatic disruption methods, the solvent extraction and carotenoids mix characterization for each pigment content [14-18].

The research information about the bioprocess presents only conventional bioreactors, stainless steel made with mechanical stirring. For the biopharmaceutical production, in line with the Good Manufacturing Practices (GMP) guidelines, the trend is to apply Single Use Bioreactors (SUB). There are already a lot of SUB configurations analysed in synthesis papers [19-22], mainly created for mammalian cells, the research for SUB for yeastcells being also atfirstresearch phases. The preliminary research done by our teams demonstrated that a yeaststrain, Rhodotorula rubra ICCF 209, can produce interesting concentrations of carotenoids mix, mainly torularhodin, function on the cultivation conditions in a classical lab bioreactor with mechanical stirring; there were also studies regarding the separation procedures $[5,23,24]$, but we are interested to further study the cultivation into SUB.

The study presents the findings regarding the optimisation of cultivation medium composition and the choice conventional versus SUB aerobic bioreactor configutations in order to increase the carotenoidic pigments concentration, but mainly torularhodin.

\section{Experimental part \\ Cultivation media and bioreactor configurations}

In a first phase the interest was to get the optimal composition of the cultivation medium for the yeast Rhodotorula rubra ICCF 209. Three variants of media were studied: (a)Medium 1 (M1) determined by previous research work [23] with the following composition: $40 \mathrm{~g} /$ $\mathrm{L}$ glucose, $1.5 \mathrm{~g} / \mathrm{L}$ yeast extract, $5 \mathrm{~g} / \mathrm{L} \mathrm{NH}_{4} \mathrm{NO}_{3^{\prime}} 1 \mathrm{~g} / \mathrm{L} \mathrm{KH}_{2} \mathrm{PO}_{4^{\prime}}$ $0.4 \mathrm{~g} / \mathrm{L} \mathrm{MgSO}{ }_{4} \times 7 \mathrm{H}_{2} \mathrm{O}, 0.4 \mathrm{~g} / \mathrm{L} \mathrm{NaCl}$. Trace elements are assumed to be used from the tap water. (b) Medium 2 (M2), where for both-growth and carotenoids production, $\mathrm{NH}_{4} \mathrm{NO}_{3}$ was replaced with $5 \mathrm{~g} / \mathrm{L} \mathrm{NH}_{4} \mathrm{H}_{2} \mathrm{PO}_{4}$ Medium $3(\mathrm{M} 3$ ), where for the same reasons $\mathrm{NH}_{4} \mathrm{NO}_{3}$ was replaced with 5 $\mathrm{g} / \mathrm{L}\left(\mathrm{NH}_{4}\right)_{2} \mathrm{SO}_{4}$, and supplemented with alanine $1 \mathrm{~g} / \mathrm{L}$ and oleic acid $1 \mathrm{~g} / \mathrm{L}$.

The experiments were carried out in three lab scale bioreactor configurations:

A.3 $\mathrm{L}$ ( $2 \mathrm{~L}$ working volume) conventional bioreactor Bioengineering $A G$.

In the bioreactor, mechanically stirred, the main parameters $\left(\mathrm{pO}_{2}\right.$ air flow rate, temperature, mixing speed, and $\mathrm{pH}$ ) are continuously controlled, and the foam level is monitored.

B. Cellexus has created an asymmetric airlift system (CellMaker), SUB, that is used in combination with a disposable asymmetric bag. It is designed for lab bioprocessing research with mammalian and microbial cell types. $\mathrm{k}$ a determinations, as the SUB manufacturer indicates, done in various conditions of gas flowrate level or liquid volume introduce a large domain of values 328.32 $h^{-1}-23.76 h^{-1}$. The configuration with $5 \mathrm{~L}$ liquid volume and gas flowrate of $7 \mathrm{~L} / \mathrm{min}$ characterized by $\mathrm{K}_{\mathrm{L}}$ a of $328.32 \mathrm{~h}^{-1}$

* email: achirvase@gmail.com; Phone: +40723540096 
is appropriate for cultivation of the yeast Rhodotorula rubra ICCF 209 with big dissolved $O_{2}$ needs.

C. SUB made by Sartorius Stedim Biotech, is a scaleable BIOSTAT CultiBagRM, with rocking platform. The manufacturer Sartorius recommends the cultivation system for microbial seed stage fermentation, due to $\mathrm{k}_{\mathrm{b}} \mathrm{a}$ values of $22.0 \mathrm{~h}^{-1}$ for full rocking speed, angle and gasflow using air and for $2 \mathrm{~L}$ bag.

\section{Analytical methods}

Cellular biomass was quantified by two methods: dried weight determination by drying the centrifuged and twice washed cell mass at $105 \pm 1^{\circ} \mathrm{C}$ until constant weight; off line optical density (OD) measurement of the cell suspension at $600 \mathrm{~nm}$ with Jenway Spectrophotometer. A calibration curve was done to directly determine the dried weight from OD measurements.

The substrate consumption was determined by analyzing the glucose concentration with Glucose Assay Kit from BioVision.

Based on the comprehensive literature a complex procedure was applied to get by HPLC analysis the already mentioned carotenoidic pigments concentrations in the analysed samples. Total pigments were extracted in acetone (with an extraction ratio of $1 \mathrm{~g}$ wet biomass $/ 5 \mathrm{~mL}$ acetone), from cellular biomass previously disrupted by three freeze-thaw cycles applied to centrifuged cultivation broth. After centrifugation $15 \mathrm{~min}$ at $10000 \mathrm{rpm}$ of the cellular debris, the collected extract was frozen overnight and then recentrifuged in the same conditions. The clear organic phase extract was recovered with a syringe and filtered through a membrane of $0.22 \mu \mathrm{m}$. For the RP-HPLC analysis appropriate dilutions were needed and samples of $20 \mu \mathrm{L}$ were analysed.

For separation and analysis of crude yeast extracts by HPLC, an HPLC system Agilent with an AgilentEclipse Plus $\mathrm{C} 18(4.6 \times 250 \mathrm{~mm} ; 5 \mu \mathrm{m})$ column, HPLC Waters 501 pump, in-line degasser equipped with Photodiode Array Detector were used. WaterEmpowered software (Version: Empower 2 software Build 2154) was applied for data acquisition and mathematical calculations.

A gradient from 70 to $100 \%$ acetone in 20 min with a flow rate of $1 \mathrm{~m} / \mathrm{min}$ at $40^{\circ} \mathrm{C}$ was applied as mobile phase. Detection was performed on a wide range from 280 to $780 \mathrm{~nm}$.

For the determination by RP-HPLC of the torularhodin and $\beta$-carotene concentrations in the obtained extracts a calibration curve for $\beta$-carotene Sigma (type II, synthetic, crystalline, $\geq 95 \%$ (HPLC)) was built using the concentrations of the standard solutions in the range 0.042 to $5.5 \mu \mathrm{g} / \mathrm{mL}$ vs. peak area measurements at $450 \mathrm{~nm}$. The calibration curve was made in MicrosoftExcel (Office 2010).

The chromatograms analysis to determine both the total carotenoids' concentration and the torularhodin concentration from each sample was presented in a previous publication [24].

\section{Results and discussions}

Three experiments were performed, representing discontinuous aerobic bioprocessing on media 1 (M1), 2 (M2), and 3 (M3), by using the three bioreactor configurations for each medium composition. General bioprocessing conditions were: strain: Rhodotorula rubra ICCF 209; inoculum of $24 \mathrm{~h}$ duration on the same medium prepared on an orbital shaker with $250 \mathrm{rpm}$; temperature $28^{\circ} \mathrm{C}$; inoculation ratio: $0.5 \%$; duration: $72 \mathrm{~h}$, except for the experiments with the medium M2, when the duration was $93 \mathrm{~h}$.
Specific parameters: A. Bioengineering bioreactor: speed, $600 \mathrm{rpm}$; air flow rate, $250 \mathrm{~L} / \mathrm{h}$; pressure, 0.5 bar. B. CellMaker: bubbled air flow rate, $10 \mathrm{~L} / \mathrm{min}$; surface air flowrate, $3 \mathrm{~L} / \mathrm{min}$; high pressure. C. BIOSTAT CultiBagRM: air flow rate, $0.4 \mathrm{~L} / \mathrm{min}$; rocking speed, 35 rocks/min; angle, 8.4ㄹ ; overpressure 11 mbar.

\section{Yeast growth \\ $1^{\text {st }}$ experiment with medium M1}

The results of OD measurements ( $600 \mathrm{~nm})$, and glucose consumption are presented in figures 1-2. Similar $p \mathrm{H}$ evolution was observed in the 3 bioreactors: initial $\mathrm{pH}$ of 4.5-4.7; slow decrese in the lag phase until 4.2; fast decrease during the exponential grow th phase until 2 , and constant level of 2 until the end. $\mathrm{pO}_{2}$ evolution measured in configurations $\mathrm{A}$ and $\mathrm{C}$ are typical and similar enough: almost $0 \%$ during $20-60 \mathrm{~h}$ period, then increase to $60 \%$ for the last period in case of $\mathrm{A}$ configuration; almost $0 \%$ during 16-40 $\mathrm{h}$, then slow increase to $40 \%$ until the end for C configuration.

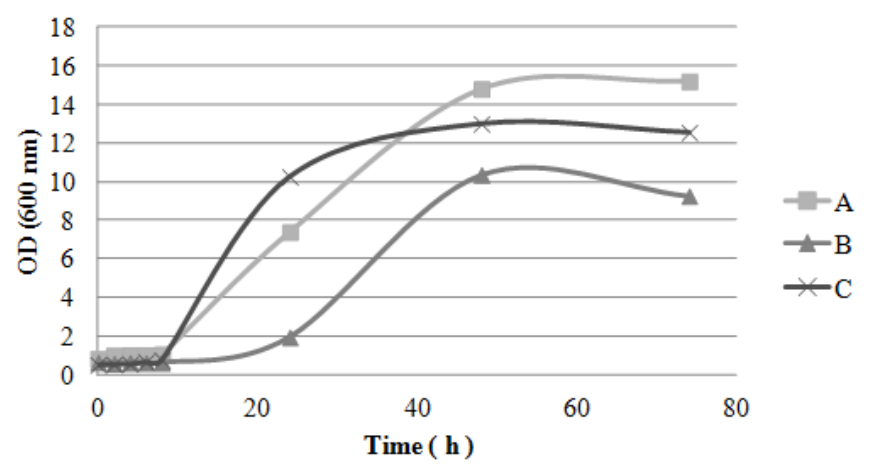

Fig. 1. Growth curves of Rhodotorula rubra ICCF 209, medium M1,

$$
\text { A-" - }\left(\mu_{\max }=0.056 \mathrm{~h}^{-1}\right), \mathrm{B}-\Delta-\left(\mu_{\max }=0.029 \mathrm{~h}^{-1}\right), \mathrm{C}\left(\mu_{\max }=0.12 \mathrm{~h}^{-1}\right)
$$

As shown in figure 1 for all cultures except for Cellexus reactor there was a lag period of about -6 h, followed by exponential growth phase until 20 to $24 \mathrm{~h}$. For the culture in the Cellexus bioreactor the lag phase was extended to $16-18 \mathrm{~h}$ and the exponential until $48 \mathrm{~h}$. Also in the case of this bioreactor a weaker cell growth (32.5\% lower) was observed.

Figure 2 presents the substrate consumption (glucose), that decreased constantly. An exception to this behavior was observed in the Cellexus bioreactor, where the

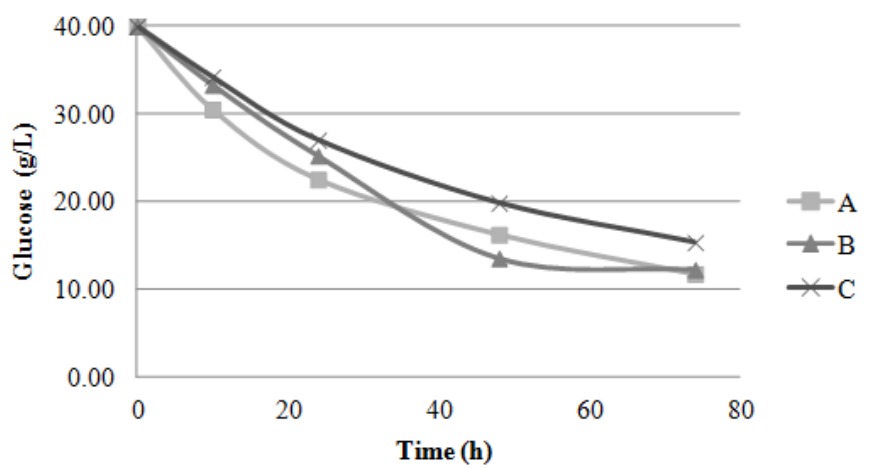

Fig. 2. Substrate consumption in time, medium $M 1$ consumption was reduced with approximately $6 \%$, due to lower cellular growth.

The maximum specific growth rate $\left(\mu_{\mathrm{m}}\right)$ ws calculated by using the exponential model. There was big difference between the three cultures. The Sartorius bioreactor (without a high intake of oxygen dissolved in culture medium) had growth rate superior $\left(0.12 \mathrm{~h}^{-1}\right)$ to the higher ventilated systems. In case of the Bioengineering 
bioreactor, even if the growth rate is lower $\left(0.056 \mathrm{~h}^{-1}\right)$, the optical density reached high values. In this case the mechanical stirring favors the oxygen and nutrients transfer from the culture medium to the cells; this did not happen in case of the Cellexus bioreactor, where the low growth rate $\left(0.029 \mathrm{~h}^{-1}\right)$ was in line with a lower optical density.

\section{$2^{\text {nd }}$ experiment with medium M2}

Again in all cases the $\mathrm{pH}$ decreased during the exponential growth phase until around $2 . \mathrm{pO}_{2}$ in the culture medium (in the two bioreactors equipped with oxygen sensor, A and C) decreased rapidlyin the first 6 to $8 \mathrm{~h}$, which corresponds to early exponential phase of growth, when oxygen consumption increases due to cell multiplication (cellular respiration). Although optical density values indicated no more cell growth, dissolved oxygen remains to a minimum by the end of culture ( $93 \mathrm{~h}$ ), which shows a high metabolic activity of the cell population. This was probably due, in comparison with the first experiment, to the replacing of the main source of inorganic nitrogen with $\mathrm{NH}_{4} \mathrm{H}_{2} \mathrm{PO}_{4}$; phosphate group represents an important element in cellular energy metabolism.

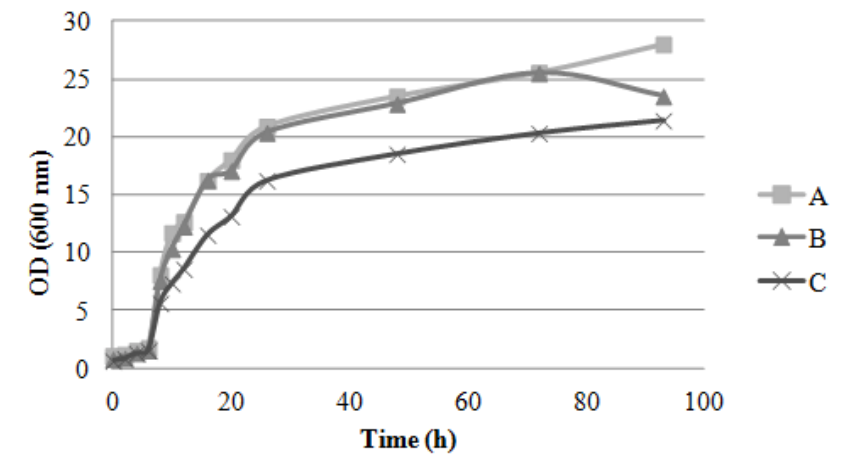

Fig. 3. Growth curves of Rhodotorula rubra ICCF 209, medium M2, A-" - $\left(\mu_{\max }=0.2 \mathrm{~h}^{-1}\right), \mathrm{B}-\boldsymbol{\Delta}-\left(\mu_{\max }=0.22 \mathrm{~h}^{-1}\right), \mathrm{C}\left(\mathrm{i}_{\max }=0.219 \mathrm{~h}^{-1}\right)$

Fig. 4. Substrate consumption in time, medium M2

Figure 3 illustrates similar growth curves of Rhodotorula rubra ICCF 209 in the 3 bioreactor configurations; the lag period was short, about 2 to $3 \mathrm{~h}$, followed by exponential grow th phase (up to $24 \mathrm{~h}$ ). It shows that the average optical density value for all the cultivations was 2 times higher than that obtained for medium 1; phosphate in cultivation medium reduces the lag phase, accelerates the cellular metabolic processes, and promotes cell proliferation.

There were no major differences between the three culture systems in terms of substrate consumption as shown in figure 4; glucose concentration decreased in the first $20 \mathrm{~h}$ of the exponential growth phase.

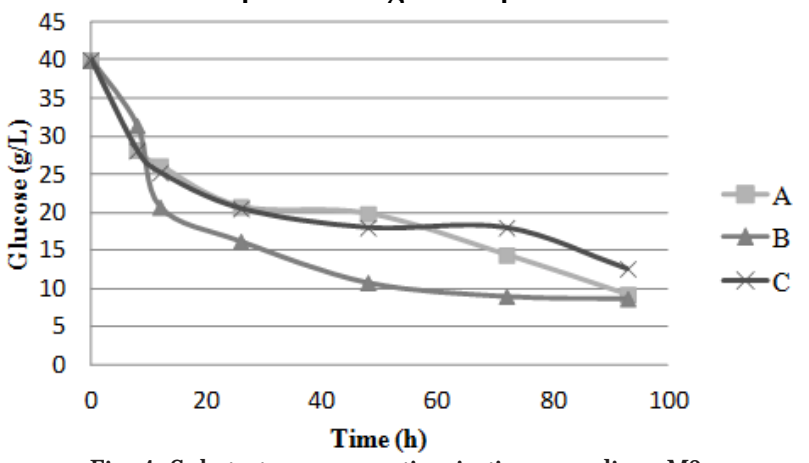

Fig. 4. Substrate consumption in time, medium M2

The maximum specific growth rates $\left(\mu_{\mathrm{m}}\right)$ had similar values in the 3 bioreactor configurations (about $0.2 \mathrm{~h}^{-1}$ ), demonstrating that the effect of additional phosphate on the cellular growth exceeded the effects of different aeration-mixing conditions conforming to each bioreactor configuration.

\section{$3^{\text {rd }}$ experiment with medium M3}

This medium formulation was amended with $\left(\mathrm{NH}_{4}\right)_{2} \mathrm{SO}_{4}$ instead of ammonium nitrate and improved by $0.1 \%$ oleic acid and $0.1 \%$ alanine.

Similar $\mathrm{pH}$ values evolutions during the exponential growth phase were coordinated with glucose consumption and cell growth.

Dissolved oxygen concentration in the culture medium in the bioreactor configurations $A$ and $C$ was characterized by a faster decrease in the first 8 to $10 \mathrm{~h}$ of culture, in correlation with the cells growth curve. As it is shown in figure 5 for all cultivation systems the yeast growth curve was similar, with short lag phase of about 3-4 $\mathrm{h}$ followed by exponential growth up to $24 \mathrm{~h}$.

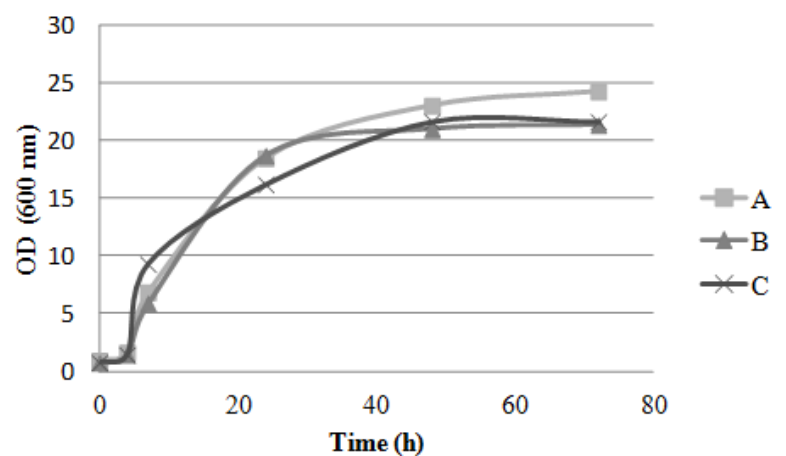

Fig. 5. Growth curves of Rhodotorula rubra ICCF 209, medium M3, A-"- $\left(\mu_{\max }=0.132 \mathrm{~h}^{-1}\right), \mathrm{B}-\mathbf{\Delta}-\left(\mu_{\max }=0.14 \mathrm{~h}^{-1}\right), \mathrm{C}\left(\mu_{\max }=0.138 \mathrm{~h}^{-1}\right)$

When introducing ammonium sulphate instead of ammonium nitrate, and supplementing with oleic acid and alanine the effects are the shortening of the lag phase and faster cell growth; final average OD is $44 \%$ higher than the average obtained on M1 medium, but lower than fig. 6).

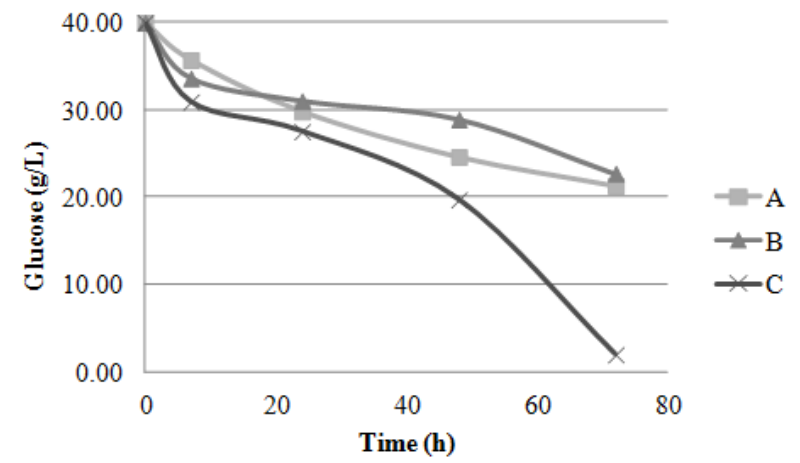

Fig. 6. Substrate consumption in time, medium M3

$\mathrm{pO}_{2}$ in the cultivation medium in both bioreactors with dissolved oxygen sensor ( $A$ and $C$ ) had fast decrease in the firsts 8-10 h, so at the beginning of the exponential growth, and there was a minimum level until the cultivation end, $72 \mathrm{~h}$. Again the improved medium composition determined an increased capacity of the cells to adapt to different cultivation conditions.

The maximum specific growth rates $\left(\mu_{\mathrm{m}}\right)$ had close values in the 3 bioreactor configurations, between 0.13 and $0.14 \mathrm{~h}^{-1}$, higher than those on the M1 medium, but lower than those on the M2 medium.

Previous results [23] indicated that the maximum concentration of total carotenoids mix with high torularhodin content was determined in the growth stationary phase, about $48 \mathrm{~h}$, confirming that these bioproducts can be considered as secondar metabolites, non growth associated. So the pigments concentrations were measured in the growth stationary phase.

The general results obtained for total carotenoids and torularhodin formation determined by acetone extraction and HPLC separation in case of the 3 experiements are presented in the figure 7 . 


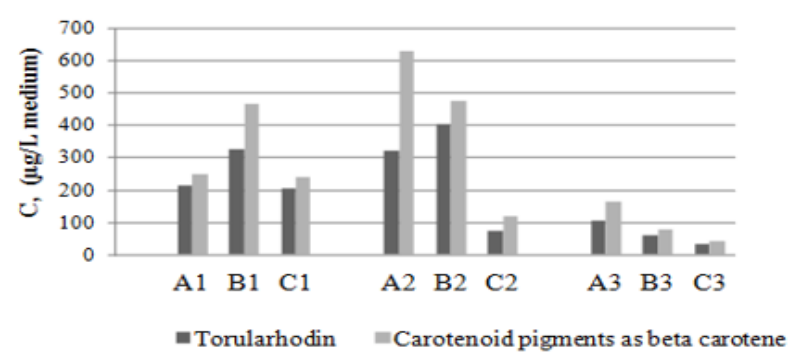

Fig. 7. The torularhodin concentration function of the experimental variants

In case of M1 medium there were similar pigments concentrations in case of $\mathrm{A}$ and $\mathrm{C}$ bioreactor configurations, butin case of Cellexus bioreactor the concentration of total carotenoids was $44.7 \%$ higher than in the other bioreactors, and similar for torularhodin concentration, with 31,7\% increase. These results are to be corellated with a smaller cellular growth in this bioreactor indicating some oxidative stress, that is stimulating the carotenoids pigments formation.

Interesting results were obtained in case of the second experiment on a medium with ammonium phosphate addition (M2). In case of bioreactors configurations A and $B$ there were the highest pigments concentrations for all experiments, but in the $\mathrm{C}$ biorector configuration the carotenoids concentration was much decreased (decreases of $70.06 \%-78.45 \%$ for total carotenoids concentrations and of $77.58-82.23 \%$ for torularhodin). The highest total carotenoids concentration was determined in the Bioengineering bioreactor, and the most increased torularhodin concentration was measured in the Cellexus bioreactor ( $84.65 \%$ form the carotenoids mix). These high pigments concentrations (considered for broth volume) are partially due to increased cellular concentrations (by comparison with M1 medium), but also to important pigments'specific concentrations (by comparison with M3 medium, where similar growth was measured). So the phosphate addition is also beneficial for pigments formation.

In case of the experiments conducted on the M3 medium, the capacity of both formation of total carotenoid pigments and torularhodin was much lower than in case of other experiments; culture medium complexity promoted cell growth, but without positive influence on the carotenoid pigment biosynthesis.

\section{Conclusions}

For further research with the same yeast it is to consider both: the medium composition with phosphate addition, but also the Single Use Bioreactor Cellexus (assymetric airlift system with disposable bag) to get the highest torularhodin concentration in the carotenoids mix.

Regarding the influences of cultivation medium composition:

-highest growth rates were obtained in M2 medium and significantly lower growth rates were determined in M1 medium;

- the same M2 medium was beneficial for carotenoids formation: in case of conventional Bioengineering bioreactor $(A)$ and SUB Cellexus (B) there were the highest pigments concentrations for all experiments, with the biggest total carotenoids concentration in the Bioengineering bioreactor, and the most increased torularhodin concentration in the Cellexus bioreactor (84.65\% from the carotenoids mix).

Regarding the influences of bioreactor configurations and the possiblity to apply SUB instead of conventional bioreactor: a) for the bioreactor Sartorius (C) although the cell growth was approached the other cultivation systems, both the total pigments concentration and the torularhodin concentration were much lower for any cultivation medium composition.

b) for the conventional bioreactor Bioengineering $(A)$ there were big enough specific growth rates, especially on M2 medium, and the biggest total carotenoids concentration on the same medium.

c) in case of the SUB Cellexus configuration the maximum specific growth rates riched the most important level on M2 medium, and there was on the same medium the most significant torularhodin formation from all experimental versions.

By integrating the conclusions determined for the two studied conditions-cultivation medium composition and bioreactor configuration: for further research with the same yeast it is to consider both: the medium composition with phosphate addition, butalso the SUB Cellexus (assymetric airlift system with disposable bag) to get the highest torularhodin concentration in the carotenoids mix.

\section{References}

1.ZOZ, L., CARVALHO, J.C., SOCCOL, V.T., et al., Brazilian Arch. Biol. and Technol., 58, nr. 2, 2015, p. 278

2.BREIEROVA, E., GREGOR, T., MAROVA, l, et al., Chem. Biodiv., 5, 2008, p. 440

3.MOLINE, M., FLORES, M.R., LiBKIND, D., et al., Photochem. Photobiol. Sci., 9, 2010, p. 1145

4.KECELI, T.M., ERGINKAYA, Z., TURKKAN, E., et al. Asian J. Chem., 25,2013, p. 42

5. TKAEOVA, J., EAPLOVA, J., KLEMPOVÁ, T., Ann. Microbiol., 67, nr. 8, 2017, p. 541

6.CARDOSO, L.A., JACKEL, S., KARP, G., et al., Biores. Techol., 200, 2016, p. 374

7.CHENG Y.T., YANG, C.F., J. Taiwan Inst. Chem. Eng., 61, 2016, p. 270 8.SAKAKI, H., NAKANISHI, T., SATONAKA, K.Y., et al., Biosci. Bioeng., 89,2000, p. 203

9.ZHANG, Z., ZHANG, X., TAN, T., Biores. Technol., 157, 2014, p. 149 10.STOILOVA, I., GEORGIEV, M., TRIFONOVA, D., et al., Eur. J . Biomed. Pharma Sci. 3, nt. 2, 2016, p. 95

11.YOO, A.Y., ALNAEELI, M., PARK, J.K., Process Biochem., 51, nr. 4, 2016, p. 463

12. DU, C., LI, Y., GUO, Y., et al., Biochem. Biophys. Res. Comm., 469, nr. 4, 2016 p. 1146

13.UNGUREANU, C., FERDES, M., Adv. Sci. Lett. 18, 2012, p. 50-53.

14.MELENDEZ-MARTINEZ, A.J., STINCO, C.M., LIU, C., et al., Food Chem, 138, 2013, p. 1341

15. WEBER, R.W.S., ANKE, H., DAVOLI, P., J. Chromatogr. A, 1145, 2007, p. 118

16. RIVERA, S.M., CANELA-GARAYOA, R., J. Chromatogr. A, 1227, 2012, p. 1

17.RAZAVI, S.H., BLANCHARD, F., MARC, I., Iran J. Chem. Chem. Eng., 25, 2006, p. 1

18. PASSARELLI, M.K., EWING, A.G., WINOGRAD, N., Anal. Chem. 85, 2013, p. 2231

19.DREHER, T., WALCARIUS, B., HUSEMANN, U., et al., Adv. Biochem. Eng. Biotechnol; 138, Ed. Eibl D. and Eibl R., Springer Verlag Berlin; 2014, p. 127

20. SHUKLA, A.A., GOTTSCHALK, U., Trends Biotechnol., 31, nr. 3, 2012, p. 147

21.SZCZYPKA, M., SPLAN, D., WOOLLS, H., et al., BioProcess International., 12, nr. 3, 2014, p. 54

22.MULLER C., NIELSEN, L., FRANDSEN, T.P., BioPharm International, 29, nr. 8, 2016, p. 18.

23.MIHALCEA, A., UNGUREANU, C., FERDES, M., et al., Rev. Chim. (Bucharest) 62, no. 6, 2011, p. 659

24.MIHALCEA, A., ONU, A., TUCUREANU, C., et al., Rev. Chim. (Bucharest). 66 no. 10, 2015, p. 1692

Mauscript received: 25.04 .2018 